\title{
Relevamiento de drogas en carros de emergencia de un hospital pediátrico. Estudio antes-después de una intervención educativa
} Assessment of emergency trolley drugs in a children's hospital.
Before and after study on an educational intervention

\author{
Dra. María I. Fernández Achával ${ }^{a}$ Dr. Luis F. Mammi ${ }^{a}$ Dr. Nicolás Fortini Cabarcosa, \\ Dr. Gonzalo G. Guiñazúa, Dra. Cecilia A. Mercedes Robledo ${ }^{a}$ Dra. Julia Dvorkin, \\ Dr. Ezequiel Monteverde ${ }^{b}$, Dra. Carolina Pascualc, Dra. Christian Elías Costa ${ }^{d} y$ \\ Dr. Eduardo O. Vainstein ${ }^{c}$
}

\section{RESUMEN}

Introducción. El abordaje de las emergencias pediátricas requiere recursos especializados y son frecuentes los errores relacionados con la medicación.

Objetivos. Describir el contenido de los carros de emergencia (CE) en un hospital pediátrico y compararloluego de una intervención educativa. Métodos. Se incluyeron CE de 9 salas de internación. Se elaboró una lista de cotejo con 30 drogas. Se relevaron los CE determinando presencia o ausencia de drogas y si las cantidades eran suficientes. Luego se realizó una intervención educativa y relevamientos 30 y 90 días posintervención.

a. Clínica Pediátrica, Comité de Docencia e Investigación, Hospital de Niños Dr. Ricardo Gutiérrez, Ciudad Autónoma de Buenos Aires (CABA).

b. Unidad de Terapia Intensiva, Hospital de Niños Ricardo Gutiérrez, CABA.

c. Unidad 5, Hospital de Niños Ricardo Gutiérrez, CABA.

d. Nefrología, Comité de Docencia e Investigación, Hospital de Niños Ricardo Gutiérrez, CABA.

Correspondencia: Dra. María I. Fernández Achával:

inesfachaval@gmail.com

Financiamiento:

Ninguno.

Conflicto de intereses:

Ninguno que declarar.

Recibido: 24-5-2019

Aceptado: 23-12-2019
Cómo citar: Fernández Achával MI, Mammi LF, Fortini Cabarcos N, Guiñazú GG, et al. Relevamiento de drogas en carros de emergencia de un hospital pediátrico. Estudio antes-después de una intervención educativa. Arch Argent Pediatr 2020;118(4):234-239.

\section{INTRODUCCIÓN}

Las emergencias que requieren reanimación con drogas son infrecuentes en pediatría. Entre ellas, el paro cardiorrespiratorio (PCR) conlleva gran morbimortalidad. En EE. UU., presenta una incidencia de 8-20/100 000 niños por año y entre el $2 \%$ y el 5,5\% de las admisiones a unidades de cuidados intensivos pediátricos. ${ }^{1-3}$ Se estima que menos del $8 \%$ de los niños atendidos por un PCR extrahospitalario sobrevivirá al evento, mientras que esta cifra asciende a un $27 \%$ si ocurre dentro del hospital. ${ }^{4}$ En los últimos años, se ha determinado una mejoría en la sobrevida de dichos pacientes, en parte, debido a las actualizaciones periódicas y a la amplia difusión de las pautas de reanimación cardiopulmonar (RCP) avanzada.

El abordaje de las emergencias pediátricas requiere recursos especializados, que incluyen equipos, drogas y personal entrenado. ${ }^{1}$ Los errores durante la RCP relacionados con el material y la medicación son frecuentes y pueden tener repercusiones en la morbimortalidad de los pacientes asistidos. ${ }^{5,6}$ Por este motivo, la aplicación debe sustentarse en un sistema organizado para que sea eficaz. ${ }^{6}$ Los centros sanitarios deben disponer del equipamiento necesario, así como de profesionales de salud adecuadamente instruidos. En este contexto, un elemento asistencial considerado indispensable es el carro de emergencias (CE). Este organiza el material utilizado durante las emergencias y se ubica en los lugares del hospital donde es más probable 
que sucedan. Debería reservarse para ser utilizado en circunstancias contempladas en los protocolos de emergencias, contando con un procedimiento de uso, reposición y mantenimiento. - $^{7-9}$

En el año 1995, la Academia Americana de Pediatría publicó guías que establecían las características de las instalaciones necesarias para la atención de emergencias pediátricas. En ellas, se definió la importancia de la organización de los recursos para brindar una respuesta óptima y oportuna, y lograr adecuados niveles de atención de pacientes críticamente enfermos. ${ }^{1}$ En 2003, se realizó una nueva recomendación, que indicaba que las drogas de uso habitual en emergencias debían estar accesibles y claramente etiquetadas utilizando algún tipo de referencia visual para el cálculo de las dosis en función del peso y / o la talla. Todos los profesionales de la salud deben conocer la ubicación de los elementos necesarios para la atención de emergencias, y se destaca la importancia de la educación continua para su correcta utilización. ${ }^{9,10}$

Si bien las guías internacionales ofrecen recomendaciones sobre el equipamiento deseable de los CE, en nuestro ámbito, su contenido no es homogéneo. A veces, sus materiales responden a otras necesidades, además de las propias de la RCP u otras emergencias, derivadas de la actividad del área donde se encuentren ubicados. ${ }^{11}$ En ocasiones, no está claramente definido a quién corresponde la responsabilidad de la reposición y del mantenimiento de su contenido. En la institución donde se realiza el estudio, no se cuenta con planillas de cotejo para la correcta provisión de drogas en la emergencia. Por todo lo expuesto, se considera de importancia realizar un estudio que describa la situación actual de los CE a fin de poner en evidencia la problemática en nuestro medio y plantear posibles soluciones.

\section{OBJETIVOS}

El objetivo primario del presente estudio fue realizar un análisis descriptivo inicial del contenido de los CE en diferentes áreas de un hospital pediátrico cotejando con una lista previamente diseñada. El objetivo secundario fue evaluar la efectividad de una intervención educativa al personal responsable del equipamiento y mantenimiento de los CE, y comparar su contenido a los 30 y 90 días posteriores.

\section{POBLACIÓN Y MÉTODOS}

Se realizó un estudio analítico cuasi experimental no controlado (antes-después) prospectivo en salas de internación de clínica dependientes del Departamento de Medicina de un hospital pediátrico de tercer nivel de atención de la Ciudad Autónoma de Buenos Aires, entre los meses de agosto de 2018 y marzo de 2019.
Sobre la base de diferentes publicaciones, se determinaron las drogas indispensables para equipar un CE, y se definió su cantidad mínima necesaria. ${ }^{1-3}$ Se consideró, para ello, la posibilidad de realizar 30 minutos de RCP avanzada según los protocolos vigentes, así como la cantidad necesaria de drogas para otras emergencias, que se consensuó con los Departamentos de Farmacia y Enfermería del Hospital y según la disponibilidad de las drogas. Con dicha información, se elaboró una lista de cotejo (Tabla 1). Se incluyeron todas las salas que contaban con CE. Se excluyó la Sala de Neurocirugía por la remodelación del servicio. Se excluyeron también aquellas salas con manejo estandarizado de la emergencia, como la Unidad de Terapia Intensiva, Terapia Intermedia y Guardia Externa.

En primera instancia, cuatro investigadores previamente entrenados relevaron los CE de 9 salas de internación, con autorización del Departamento de Medicina, pero sin previo aviso a los jefes de Enfermería y Clínica de cada sala: 4 Salas de Clínica Pediátrica y 5 de Clínica con Orientación (Hepatología, Neumonología, Nefrología y 2 de Infectología), utilizando la lista de cotejo previamente diseñada. Cada sala disponía de un único $C E$, cuya confección, mantenimiento y verificación se encontraba a cargo de los jefes de Enfermería. Se determinó, para cada CE, la presencia o ausencia de las drogas. En caso de estar presentes, se constató, además, si se encontraban vencidas o en cantidad suficiente o insuficiente.

Finalizado el relevamiento inicial de datos, se procedió a la capacitación del personal responsable de los CE de cada sala evaluada. Consistió en dos encuentros presenciales de 1 hora cada uno, en los que se expuso la evidencia de la importancia de contar con el material adecuado para llevar a cabo RCP básica y avanzada de calidad, así como otras emergencias. Se entregó la planilla de cotejo y se entrenó al personal en su uso. A los 30 y 90 días posintervención, se realizaron nuevos relevamientos de todos los CE en horarios similares a los de la primera medición. Las visitas no se anticiparon al personal médico ni de Enfermería de cada sala para no sesgar los resultados.

Antes de la recolección de datos, se elaboró un manual estandarizado de procedimientos. En él, se detallaron la codificación de las planillas, el correcto ingreso de datos y cómo consignar aquella información ausente o imprecisa. Las drogas fueron divididas en 9 categorías para ser analizadas (Tabla 2). Se realizó la doble entrada de datos, en forma ciega, por dos operadores entrenados. Se compararon los datos ingresados y no se evidenciaron diferencias.

En primer lugar, se realizó un análisis descriptivo. Se determinó, por cada sala de 
internación y para cada una de las tres mediciones, el porcentaje de ítems correctos con su intervalo de confianza (IC) del $95 \%$ y, además, las razones por las cuales los ítems fueron considerados incorrectos. Posteriormente, se compararon las mediciones y se utilizó el test de McNemar para datos emparejados para el cálculo de las medidas de efecto y el nivel de significancia de las diferencias halladas. Se utilizó el paquete estadístico $R$.

Antes de la realización de este estudio, se solicitó la aprobación de los jefes de unidades participantes, del Jefe del Departamento de Medicina y del Comité de Ética y del Comité de Docencia e Investigación del Hospital. Asimismo, se resguardó la confidencialidad de todos los datos recabados, que fueron codificados en las planillas de recolección de datos.

\section{RESULTADOS}

La primera medición arrojó una baja proporción global de drogas adecuadas (es decir, ni vencidas ni insuficientes ni ausentes) del $43,9 \%$ (IC $95 \%: 38,4-49,4$ ) con una amplitud entre CE

TABLA 1. Lista de cotejo de fármacos del carro de emergencias

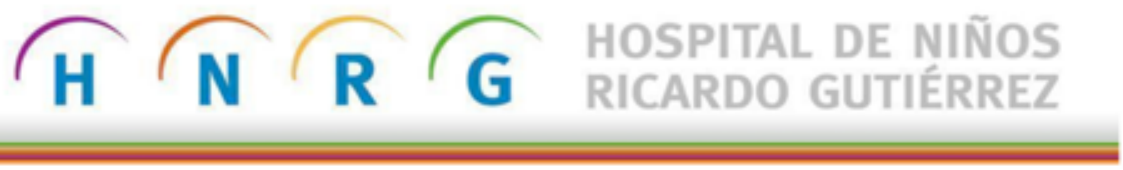

LISTA DE COTEJO DE FÁRMACOS DEL CARRO DE PARO

\begin{tabular}{|c|c|c|c|}
\hline \multicolumn{4}{|l|}{ UNIDAD/SERVICIO: } \\
\hline \multicolumn{4}{|l|}{ FECHA DE CHEQUEO: } \\
\hline \multicolumn{4}{|l|}{ PRECINTO N.\%CÓDIGO: } \\
\hline \multicolumn{4}{|c|}{$\begin{array}{l}\text { Se debe recordar corroborar la fecha de vencimiento de cada ampolla. } \\
\text { Se deben descartar aquellas vencidas y/o que vencerán en el próximo mes. }\end{array}$} \\
\hline Fármacos & Cantidad mínima necesaria & $\begin{array}{c}\text { Fecha de } \\
\text { vencimiento }\end{array}$ & Cotejo \\
\hline Adenosina & 2 ampollas & & \\
\hline Amiodarona & 2 ampollas & & \\
\hline Adrenalina & 10 ampollas & & \\
\hline Atropina & 3 ampollas & & \\
\hline Bicarbonato de sodio & 100 meq $\times 2$ & & \\
\hline Bloqueantes musculares (Vecuronio) & $10 \mathrm{mg}$ & & \\
\hline Cloruro de potasio $3 \mathrm{M}$ & 4 ampollas & & \\
\hline Dopamina & 5 ampollas & & \\
\hline Fenitoina = difenhilhidantoina & 15 ampollas & & \\
\hline Gluconato de calcio al $10 \%$ & 2 ampollas & & \\
\hline Lidocaina & 3 ampollas & & \\
\hline Naloxona & 5 ampollas & & \\
\hline Sulfato de magnesio al $25 \%$ & 2 ampollas & & \\
\hline Fentanilo & 2 ampollas & & \\
\hline Diazepam & 2 ampollas & & \\
\hline Difenhidramina & 2 ampollas & & \\
\hline $\begin{array}{l}\text { Antipiréticos endovenosos: } \\
\text { dipirona/ibuprofeno }\end{array}$ & 2 ampollas & & \\
\hline Flumazenil & 2 ampollas & & \\
\hline Furosemida & 5 ampollas & & \\
\hline Haloperidol & 2 ampollas & & \\
\hline $\begin{array}{l}\text { Hidrocortisona o dexametasona o } \\
\text { metilprednisolona }\end{array}$ & 2 ampollas & & \\
\hline Insulina & 1 ampolla en HELADERA & & \\
\hline Ketamina & 1 ampolla & & \\
\hline Lorazepam & 4 ampollas en HELADERA & & \\
\hline Midazolam & 3 ampollas & & \\
\hline Solución dextrosada & 2 sachets & & \\
\hline Solución fisiológica de $500 \mathrm{ml}$ & 2 sachets & & \\
\hline Solución fisiológica & 10 ampollas & & \\
\hline Agua destilada & 10 ampollas & & \\
\hline
\end{tabular}

Para confeccionar esta lista de cotejo, se determinaron las drogas indispensables para equipar un CE y se definió su cantidad mínima necesaria. Fue elaborada sobre la base de la bibliografía, en consenso con el Departamento de Farmacia y el de Enfermería del Hospital según la disponibilidad y la accesibilidad de las drogas.

meq: miliequivalentes; ml: mililitros; M: molar. 
del $29 \%$ al 54,8 \% y, entre grupos de drogas, del $15 \%$ (diuréticos) al $85 \%$ (antídotos) (Figura 1). La segunda medición a los 30 días mostró una proporción correcta del 70,3\% (IC $95 \%$ : 65,2$75,4)$ con aún mayor variación entre los CE (del

TABla 2. Agrupamiento de las drogas del carro de emergencias según su utilidad en la emergencia

\begin{tabular}{|c|c|}
\hline Grupo & Droga \\
\hline Antiarrítmicos & $\begin{array}{l}\text { Amiodarona } \\
\text { Atropina } \\
\text { Lidocaína }\end{array}$ \\
\hline Anticonvulsivantes & $\begin{array}{l}\text { Diazepam } \\
\text { Difenilhidantoína } \\
\text { Fenobarbital } \\
\text { Lorazepam }\end{array}$ \\
\hline Antídotos & $\begin{array}{l}\text { Flumazenil } \\
\text { Naloxona }\end{array}$ \\
\hline Diuréticos & $\begin{array}{l}\text { Furosemida } \\
\text { Manitol }\end{array}$ \\
\hline Drogas de anafilaxia & $\begin{array}{l}\text { Difenhidramina } \\
\text { Hidrocortisona/dexametasona }\end{array}$ \\
\hline Drogas de IOT & $\begin{array}{l}\text { Fentanilo } \\
\text { Ketamina } \\
\text { Midazolam } \\
\text { Vecuronio }\end{array}$ \\
\hline $\begin{array}{l}\text { Soluciones } \\
\text { hidroelectrolíticas }\end{array}$ & $\begin{array}{l}\text { Bicarbonato } \\
\text { Cloruro de potasio } \\
\text { Gluconato de calcio } \\
\text { Sulfato de magnesio } \\
\text { Ampollas de solución fisiológica } \\
\text { Ampollas de agua destilada } \\
\text { Solución Ringer lactato } \\
\text { Solución fisiológica } \times 500 \mathrm{ml}\end{array}$ \\
\hline Vasopresores & $\begin{array}{l}\text { Adrenalina } \\
\text { Dopamina }\end{array}$ \\
\hline Otras & $\begin{array}{l}\text { Dipirona } \\
\text { Insulina } \\
\text { Solución dextrosada }\end{array}$ \\
\hline
\end{tabular}

IOT: intubación orotraqueal.
$51,6 \%$ al 93,5 \%) y, entre grupos de drogas, del $50 \%$ (vasopresores) al $95 \%$ (antídotos). La tercera medición a los 90 días mostró resultados similares a los de la segunda: correctos del 69,4\% (IC 95\%: 64,3-74,5), con una variación también similar entre CE (del 51,6 \% al 93,5\%) y, entre drogas, del $45 \%$ (antiarrítmicos) y del $90 \%$ (antídotos). Las razones por las cuales los ítems se consideraron inadecuados en las tres mediciones se muestran en la Tabla 3.

La comparación entre la primera y la segunda medición mostró una mejoría en todos los CE (rango del 3,2\% al 45,1\%), con una diferencia entre ambas mediciones estadísticamente significativa: odds ratio (OR) 3,73 (IC $95 \%$ : 2,5$5,6), p<0,001$. Se evaluó si los CE con mayor proporción inicial de ítems correctos presentaban una proporción de mejoría superior a través de la correlación entre proporción de correctos y tamaño del cambio, y se observó un índice $\mathrm{R}$ de Spearman de -0,29.

La comparación entre la segunda y la tercera medición mostró resultados similares: $O R$ 1,1 (IC95 \%: 0,7-1,8), p=0,71, con descenso de ítems correctos en tres CE (rango del 3,2\% al $9,7 \%$ ), sin cambios en uno y con mejoría en los cinco restantes (rango del 3,2\% al 22,6\%). La evaluación entre los dos extremos (primera y tercera medición) mostró una mejoría significativa y sostenida durante el período evaluado: un CE se mantuvo estable y el resto mostró mejoría (rango: del 19,4 \% al 61,3\%) con un OR 3,55 (IC95 \%: 2,45,3), $\mathrm{p}<0,001$ (Figura 2).

\section{DISCUSIÓN}

De acuerdo con la Agencia de Investigación y Calidad en Salud de EE. UU., la mejora en la calidad de atención se alcanza a través del pensamiento sistemático, el análisis de datos y el

Figura 1. Evaluación de ítems correctos en la primera medición. Porcentaje de drogas adecuadas según categoría

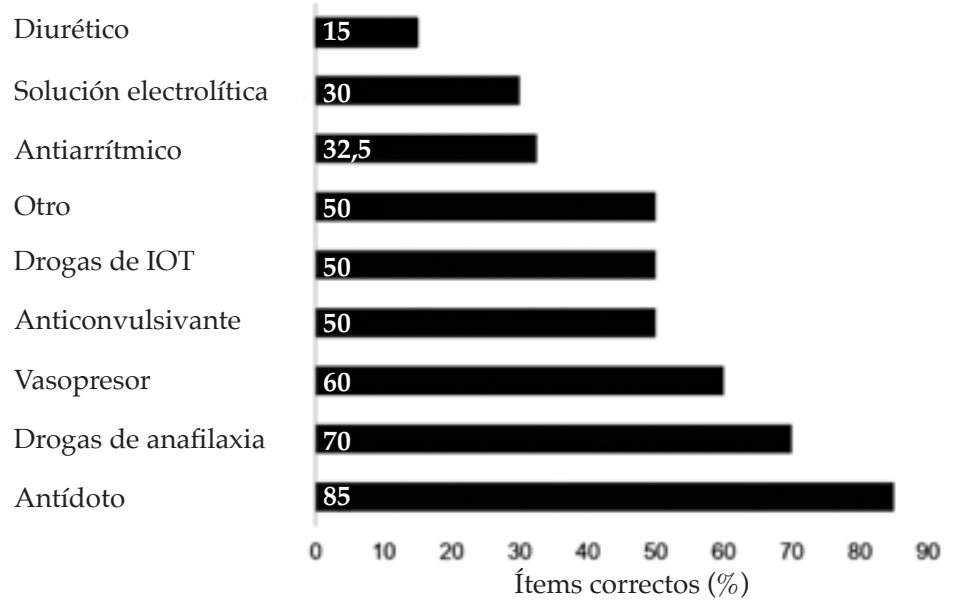

IOT: intubación orotraqueal. 
trabajo en equipo para lograr mejores resultados, mejores procesos y reducir los errores que puedan afectar a los pacientes y sus familias. ${ }^{12,13}$ Una de las dimensiones de la calidad es la seguridad, entendida como la reducción del riesgo de daños innecesarios hasta un mínimo aceptable. ${ }^{14}$

Existen varios estudios que buscaron analizar los errores más comunes ocurridos durante las emergencias. ${ }^{6,16-19}$ Es evidente que, ante una situación estresante, como un PCR, puedan ocurrir errores en la administración de fármacos y un inadecuado suministro podría ser el primer eslabón de una cadena de errores. Es por ello que un enfoque estandarizado genera un ambiente más seguro, especialmente, cuando el usuario no se encuentra familiarizado con el manejo. ${ }^{19}$ En el presente estudio, se pudo evidenciar una considerable mejora global en la adecuación de

Tabla 3. Clasificación de ítems incorrectos. Diferencias entre los tres tiempos de medición para los items incorrectos clasificados como ausentes, insuficientes y vencidos

\begin{tabular}{lccc}
\hline & $\begin{array}{c}\text { Medición basal } \\
\mathbf{n}(\%)\end{array}$ & $\begin{array}{c}\text { 30 días } \\
\mathbf{n}(\%)\end{array}$ & $\begin{array}{c}\mathbf{9 0} \text { días } \\
\mathbf{n}(\%)\end{array}$ \\
\hline Ausencia & $107(62,6)$ & $38(46,3)$ & $24(43,6)$ \\
Insuficiente & $37(21,6)$ & $25(30,5)$ & $27(49,1)$ \\
Vencido & $27(15,8)$ & $19(23,2)$ & $4(7,3)$ \\
Total & $171(100)$ & $82(100)$ & $55(100)$ \\
\hline
\end{tabular}

n: número de mediciones. las drogas de los CE luego de una intervención educativa. Todos los CE de las salas analizadas mostraron mejoras significativas, aunque los resultados no fueron homogéneos entre las distintas salas de internación. Es importante destacar que los resultados del relevamiento se mantuvieron en el período evaluado, y no se encontraron diferencias significativas entre la segunda y la tercera mediciones. Adicionalmente, se observó que las salas que contaban con mayor proporción de drogas adecuadas fueron las que menos modificaron su contenido inicial.

Fierro Rosón y col., evaluaron el contenido y funcionamiento de los CE en un hospital español y describieron deficiencias que podrían comprometer o dificultar la eficacia de la RCP: fueron minoría los CE adecuadamente equipados. ${ }^{12}$ La existencia de errores en el manejo de emergencias, como la RCP, debido a problemas en la utilización de recursos puede generar un evento centinela (entendido como aquel que genera un evento adverso grave). ${ }^{13-15}$ Este tipo de evento evidencia una debilidad en el sistema que requiere un análisis y reparación inmediatos. Debido a ello, es relevante realizar intervenciones que contemplen esta problemática en nuestro medio.

Además, se debe considerar que es fundamental que los servicios de salud puedan

FIGURA 2. Comparación entre las tres mediciones. Proporción de items correctos sobre el total para las tres mediciones. Odds ratio de la diferencia entre la primera y la segunda medición y entre la segunda y la tercera medición

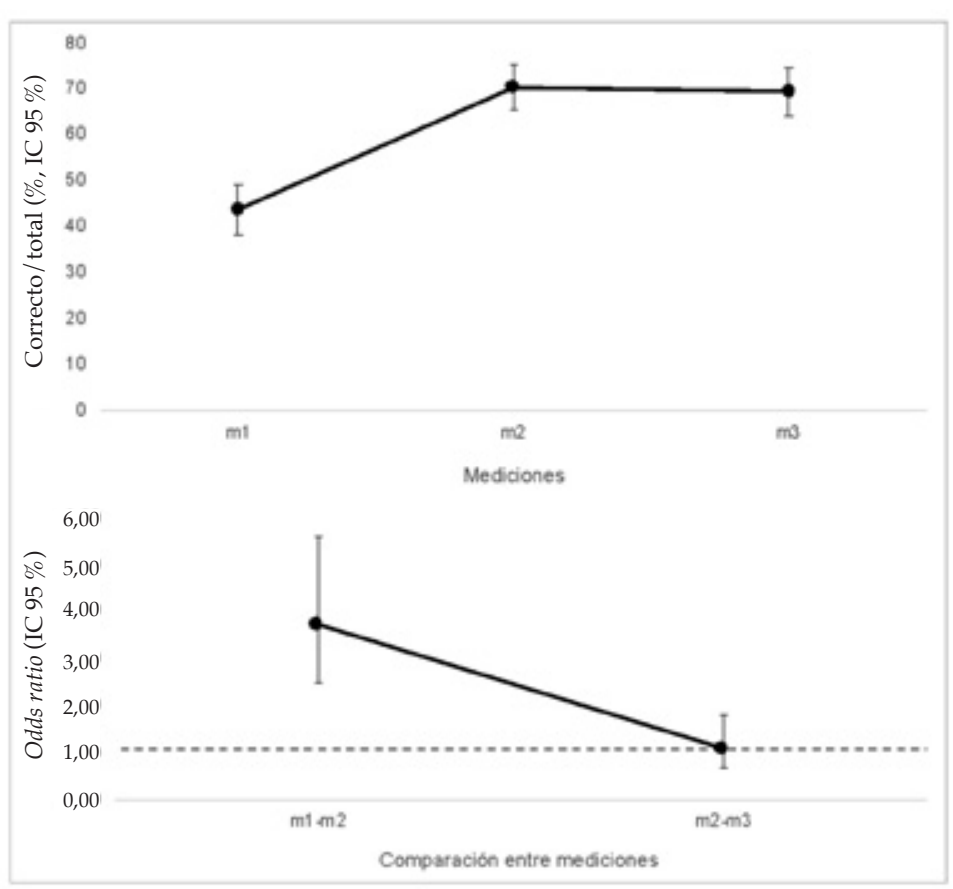

IC: intervalo de confianza. 
contar con personal entrenado periódicamente, incluso en las salas donde hay baja frecuencia de RCP u otras emergencias. Según las recomendaciones internacionales, uno de los pasos en la mejora de la supervivencia consiste en la posibilidad de contar con personal capacitado de manera continua, ya que las capacidades adquiridas disminuyen luego de meses de formación. ${ }^{2}$

El trabajo presentó algunas debilidades. El contenido de los CE analizado fue exclusivamente de drogas. Otros múltiples materiales, como aquellos dispositivos necesarios para el manejo de la vía aérea o desfibriladores, son parte imprescindible del CE. No se han analizado dichos elementos, dado que, por el formato de nuestros CE (valijas pequeñas o carros con escasos cajones y estantes no sellados), muchos se encuentran en las salas de procedimientos o contiguos a los $\mathrm{CE}$, pero no en los CE. Es nuestro propósito incluirlos en futuras mediciones. Además, dada la heterogeneidad de los CE, la distribución de las drogas dentro de los respectivos compartimentos no se pudo evaluar.

Se conoce la disparidad en los protocolos para el equipamiento de CE de las distintas instituciones privadas y públicas de nuestra ciudad, así como la ausencia de normas en ellas. Esto puede debilitar la validez externa de nuestro estudio. Además, áreas con mayor frecuencia de RCP, como el Departamento de Urgencias, la Unidad de Cuidados Intensivos o la Unidad de Cuidados Intermedios, fueron excluidas de este análisis, debido a diferencias estructurales con el resto de las salas.

Es una fortaleza de este trabajo haber detectado una falencia importante en la actividad asistencial, como lo es el inadecuado equipamiento de los CE. Asimismo, haber demostrado que la protocolización del armado de los CE, junto con una intervención educativa, puede lograr mejoras significativas y sostenidas. Se propone continuar con las mediciones en el tiempo y ampliar las intervenciones a otras áreas del Hospital buscando homogeneizar el contenido de los CE.

\section{CONCLUSIONES}

En la medición inicial, se evidenció un bajo nivel de adecuación de las drogas de los CE. El nivel fue heterogéneo entre las distintas salas de internación. Luego de la intervención, la adecuación mejoró significativamente y se mantuvo durante el período evaluado.

\section{REFERENCIAS}

1. Committee on Pediatric Emergency Medicine. American Academy of Pediatrics. Guidelines for Pediatric Emergency Care Facilities. Pediatrics. 1995; 96(3 Pt 1):526-37.
2. MonsieursKG,NolanJP, BossaertLL, GreifR, etal.European Resuscitation Council Guidelines for Resuscitation 2015 Section 1. Executive summary. Resuscitation. 2015; 95:1-80.

3. NeumarRW, ShusterCM, Callaway CW, GentLM, etal.Part 1: Executive Summary: 2015 American Heart Association Guidelines Update for Cardiopulmonary Resuscitation and Emergency Cardiovascular Care. Circulation. 2015; 132(18 Suppl 2):s315-67.

4. Nadkarni VM, Larkin GL, Peberdy MA, Carey SM, et al. First documented rhythm and clinical outcome from inhospital cardiac arrest among children and adults. JAMA. 2006; 295(1):50-7.

5. Meaney PA, Nadkarni VM, Cook EF, Testa M, et al. Higher survival rates among younger patients after pediatric intensive care unit cardiac arrests. Pediatrics. 2006; 118(6):2424-33.

6. FlanneryAH,ParliSE.MedicationErrorsinCardiopulmonary Arrest and Code-Related Situations. Am J Crit Care. 2016; 25(1):12-20.

7. Regalado Becerra CA, Segura Vega J, Órnelas Aguirre JM. Evaluación de conocimientos y equipamiento en los carros rojos para la reanimación cardiopulmonar en una unidad de tercer nivel de atención. Medicrit. 2008; 5(2):63-73.

8. López-Herce Cid J, Rodríguez Núñez A, Carrillo Álvarez A, Zeballos Sarrato G, et al. Recomendaciones de expertos sobre material del carro y mochila de reanimación cardiopulmonar pediátrica y neonatal. An Pediatr (Barc). 2018; 88(3):173. e1-173.e7.

9. Bowden T, SmithD. An overview of adult cardiopulmonary resuscitation equipment. Nurs Stand. 2017; 31(23):54-63.

10. Castillo J, García-Guasch R. Organización y enseñanza de la reanimación cardiopulmonar. Rev Esp Anestesiol Reanim. 1999; 46:183-5.

11. Sigrest TD; American Academy of Pediatrics Committee on Hospital Care. Facilities and equipment for the care of pediatric patients in a community hospital. Pediatrics. 2003; 111(5 Pt 1):1120-2.

12. Fierro Rosón J, Ruiz Bailén M, Peinado Rodríguez J, Ramos cuadraJA, et al. Evaluación del contenido y funcionamiento delos carros dereanimación cardiopulmonar deun hospital. Med Intensiva. 2003; 27(6):399-403.

13. Donabedian A. The Definition of Quality and Approaches to its Assessment. Explorations in Quality Assessment and Monitoring, Vol. 1. Chicago, IL: Health Administration Press; 1980.

14. Organización Mundial de la Salud, Patient Safety. Más que palabras. MarcoConceptual dela Clasificación Internacional para la Seguridad del Paciente Versión 1.1. Informe técnico 2009. [Acceso: 26 de abril de 2019]. Disponible en: www. who.int/ patientsafety/implementation/taxonomy/icps_ technical_report_es.pdf.

15. Joint Commission International. Standard QPS.7: The hospital uses a defined process for identifying and managing sentinel events. En: Joint Commission International Accreditation standards for hospitals. 5th ed. Oakbrook Terrace, IL: Joint Commission Resources: 2013;145.

16. Subcomisión de Calidad en Salud y Seguridad del Paciente Sociedad Argentina de Pediatría, Sabio Paz V, Panattieri N, Godio C, et al. Seguridad del paciente: glosario. Arch Argent Pediatr. 2015; 113(5):469-72.

17. Agarwal S, Swanson S, Murphy A, Yaeger K, et al. Comparing the utility of a standard pediatric resuscitation card with a pediatric resuscitation cart based on the Broselow tape: a randomized, controlled, crossover trial involving simulated resuscitation scenarios. Pediatrics. 2005; 116(3):e326-33.

18. Bergon-Sendin E, Pérez-Grande MdC, Lora-Pablos D, Melgar-Bonis A, et al. Auditorías de seguridad en tiempo real en una unidad neonatal. An Pediatr (Barc). 2017; 87(3):148-54.

19. Griffiths SE, Boleat E, Goodwin A, Sheikh A, et al. Efficacy of paediatric anaesthetic trolleys: A call for a basic standard and layout. Int J Risk Saf Med. 2015; 27(4):201-7. 